\title{
Efeitos de um programa de educação postural para crianças e adolescentes após oito meses de seu término
}

\author{
Effects of a postural program for children and adolescents eight months after its end
}

\author{
Cláudia Tarragô Candotti ${ }^{1}$, Silvia Elisandra B. Nunes², Matias Noll ${ }^{3}$, Kate de Freitas ${ }^{2}$, Carla Harzheim Macedo²
}

\section{RESUMO}

Objetivo: Avaliar os efeitos de um Programa de Educação Postural (PEP) para crianças e adolescentes oito meses após seu término.

Métodos: Estudo experimental com 34 participantes divididos em Grupo Controle (GC) e Grupo Experimental (GE). Somente os integrantes do GE participaram do PEP. Os 34 participantes foram submetidos - no início, no término e oito meses após o término do PEP - a três procedimentos de avaliação: (1) postura estática por meio de fotografia; (2) postura dinâmica, por filmagem da execução de atividades da vida diária (AVDs); e (3) questionário sobre os conhecimentos teóricos da coluna vertebral. Para verificar as diferenças entre o GC e o GE e entre as etapas de avaliação, foram utilizados os testes de Mann-Whitney e de Wilcoxon para os escores das AVDs e do questionário e o qui-quadrado para comparar a frequência de alterações posturais.

Resultados: O PEP, quando avaliado imediatamente após seu término, promoveu efeito positivo apenas no conhecimento teórico e na postura das AVDs. Entretanto, o efeito positivo do PEP não foi estendido ao período de follow-up, após oito meses do término do programa.

Conclusões: Especula-se que o conhecimento da importância da boa postura durante as AVDs não foi efetivamente incorporado aos hábitos das crianças e dos adolescentes.

Palavras-chave: postura; estudantes; educação em saúde.

\section{ABSTRACT}

Objective: To evaluate the effects of a Postural Education Program (PEP) for children and adolescents eight months after its completion.

Methods: 34 subjects were assigned to a Control Group (CG) or to an Experimental Group (EG). Only the EG participated in the PEP, but all the subjects were evaluated before, during and eight months after finishing the PEP. The assessment used three procedures: (1) static posture by photography; (2) dynamic posture by recording the execution of everyday activities (EAs); and (3) answering a questionnaire designed to evaluate the theoretical knowledge about the spine. In order to verify the differences between CG and EG and between the three studied periods the following tests were used: Mann-Whitney and Wilcoxon for comparing the scores of EAs and questionnaire, and chi-square to analyze the frequency of postural changes in CG and EG.

Results: When evaluated immediately after its completion, the PEP had a positive effect on the posture of EAs and on the knowledge about the spine. The positive effect of the PEP was reduced eight months after its completion.

Conclusions: It can be speculated that the awareness of the importance of good posture during EAs was not effectively incorporated into the habits of the studied group.

Key-words: posture; students; health education.
Instituição: Universidade Federal do Rio Grande do Sul (UFRGS), Porto Alegre, RS, Brasil

'Doutora em Ciências do Movimento Humano pelo Programa de PósGraduação em Ciências do Movimento Humano da Escola de Educação Física da UFRGS; Professora do Curso de Fisioterapia da UFRGS, Porto Alegre, RS, Brasil

2Graduada em Educação Física pela Universidade do Vale do Rio dos Sinos (Unisinos); São Leopoldo, RS, Brasil

${ }^{3}$ Mestrando em Ciências do Movimento Humano pelo Programa de PósGraduação em Ciências do Movimento Humano da Escola de Educação Física da UFRGS, Porto Alegre, RS, Brasil
Endereço para correspondência:

Cláudia Tarragô Candotti

Rua Fernando Osório, 1.887

CEP 91720-330 - Porto Alegre/RS

E-mail: claudia.candotti@ufrgs.br

Conflito de interesse: nada a declarar

Recebido em: 3/8/2010

Aprovado em: 2/2/2011 


\section{Introdução}

Profissionais ligados à área da saúde vêm buscando soluções para atenuar problemas relacionados à má postura, os quais originam, não raramente, dores que conduzem à diminuição da capacidade física e psíquicas. Nas últimas décadas, em diversos locais do mundo ${ }^{(1-9)}$, uma opção para atenuar os problemas de má postura são as Escolas Posturais, que, independentemente da sua concepção teórica, têm o objetivo de modificar as más atitudes diárias que conduzem a danos na coluna vertebral ${ }^{(3,10)}$. Desse modo, as Escolas Posturais podem ser oferecidas a públicos distintos, como, por exemplo, terceira idade, crianças, adolescentes e jovens ${ }^{(1,4-8,11-14)}$, desde que seus conteúdos teóricos e práticos sejam adaptados às diferentes necessidades.

A literatura tem referenciado que participantes de programas de Escola Postural, em diferentes faixas etárias, tendem a modificar positivamente sua postura durante as atividades da vida diária (AVDs), bem como seus conhecimentos teóricos sobre a coluna vertebral ${ }^{(6-8,10,15-18)}$. Entretanto, os relatos são escassos no que tange à questão de os ensinamentos sobre a postura serem incorporados e mantidos nos hábitos diários dos indivíduos participantes de uma dessas escolas. Desse modo, acredita-se serem necessários estudos que avaliem se os efeitos de uma Escola Postural persistem para além dos muros dos próprios programas. Além disso, é importante conhecer esses efeitos em realidades distintas, como, por exemplo, crianças e adolescentes.

Considerando-se que o ensino fundamental e os projetos sociais dirigidos a crianças e pubescentes adquirem grande responsabilidade do ponto de vista da promoção da saúde ${ }^{(8,12-15,17)}$ e pressupondo-se que, durante a fase de desenvolvimento, crianças e adolescentes adquirirem hábitos positivos de postura e tendem a mantê-los ao longo da vida, o objetivo deste estudo foi avaliar os efeitos de um Programa de Educação Postural (PEP) para as crianças e adolescentes participantes de um projeto social, oito meses após o seu término. Especulou-se que, após oito meses do término do PEP, as crianças e os adolescentes seriam capazes de conhecer e identificar a coluna vertebral, suas partes e funções, bem como manter as suas curvaturas naturais, seja durante a postura estática ou durante as AVDs.

\section{Método}

A amostra foi composta por 34 participantes do Projeto Escolinhas Integradas Airton Senna (PEI) (vinculado à
Universidade do Vale do Rio dos Sinos), de ambos os sexos, divididos em dois grupos: Controle (GC) e Experimental (GE), cada um composto por sete crianças e dez adolescentes. O cálculo amostral determinou um número mínimo de 28 indivíduos, para um nível de significância de 5\%, com um poder da amostra de $80 \%$ para diferenças de quatro unidades entre os escores, assumindo-se um desvio padrão comum de 3,5. A idade média das crianças foi de $10,5 \pm 0,8$ anos e dos adolescentes, de 13,2 $\pm 1,0$ anos. Dentre o total de participantes do PEI ( $\mathrm{n}=58$ ), os estudantes que compuseram a amostra foram sorteados para integrarem o GC (turno manhã) e o GE (turno tarde).

O critério de inclusão em cada grupo foi frequentar o PEI no turno inverso à escola formal e estar fisicamente apto para realizar atividade física. O critério de exclusão foi apresentar fraturas ou utilizar órteses (muletas). Todos os estudantes participaram voluntariamente deste estudo após o consentimento dos pais ou responsáveis. O estudo foi aprovado pelo Comitê de Ética em Pesquisa da Universidade Federal do Rio Grande do Sul (UFRGS) e respeitou a Resolução 196/96 do Conselho Nacional de Saúde.

Foram realizados três procedimentos de avaliação: (1) postura estática; (2) postura dinâmica e (3) conhecimentos teóricos sobre a coluna vertebral, nos períodos de pré-determinados. O PEP teve duração de 45 dias e o estudo avaliou os desfechos em três períodos: pré-intervenção, imediatamente pós-intervenção e oito meses depois do final da intervenção (follow-up).

O PEP consistiu em uma Escola Postural com metodologia adaptada de Souza ${ }^{(19)}$ no que diz respeito à escolha das AVDs e à linguagem empregada para comunicar-se com as crianças e adolescentes. Por exemplo, na Aula 3, referente "à posição sentada", enfatizou-se a posição sentada na escola e à frente do computador. Já na Aula 5 , referente a “permanecer em pé e caminhar corretamente", enfatizou-se o transporte do material escolar. O Quadro 1 apresenta, de forma esquemática, as etapas do experimento, bem como explicita cada aula com seu tema, objetivos e AVDs. As aulas foram desenvolvidas duas vezes por semana, com duração de uma hora cada, apenas com o GE. As crianças do GC não receberam qualquer informação ou mantiveram contato com o Programa. Isso só foi possível porque as crianças de ambos os grupos eram participantes do PEI em turnos distintos.

$\mathrm{Na}$ avaliação da postura estática, as crianças e os adolescentes foram avaliados de forma individual, utilizando vestimenta adequada, na posição de perfil direito, por meio 
de um posturógrafo e de um fio de prumo. O estudante foi posicionado no posturógrafo, sendo o fio de prumo ajustado segundo Kendall et a ${ }^{(20)}$. Após o posicionamento, solicitou-se que adotassem a postura que considerassem correta e, nesse momento, eram fotografados.

Para a análise da postura estática, as fotografias dos GC e GE, nos períodos pré, pós-experimento e follow-up, foram misturadas e entregues a um especialista em postura, sem contato com as crianças e os adolescentes, para que o mesmo realizasse a avaliação. Esse procedimento foi necessário para evitar um viés na avaliação da postura estática. O especialista avaliava e indicava por onde passava o fio de prumo, focalizando o ombro e o lóbulo da orelha, em relação à postura padrão, o que permitiu avaliar a postura do ombro (protruso, retraído ou normal) e a posição da cabeça (anteriorizada, em extensão axial ou normal). Foram consideradas alterações posturais do ombro se fosse assinalado "protruso" ou "retraído" e alterações posturais da cabeça quando era anotado "anteriorizada" ou "em extensão axial". Para a análise da postura estática, foram contabilizados apenas o número total de casos em que ocorreu alguma alteração postural, seja no ombro ou na cabeça, nos períodos de pré, pós-experimento e follow-up.

A avaliação dinâmica da postura consistiu na filmagem individual durante um circuito de AVDs ${ }^{(21)}$ : (1) ato de sentar em um banquinho; (2) posição sentada no banco; (3) pegar um objeto pesado e um leve do solo; (4) carregar esses objetos até uma mesa; (5) recolocar os objetos no solo e (6) posição sentada na cadeira escrevendo em uma mesa.

A análise da postura dinâmica foi realizada a partir do vídeo, de acordo com Rocha e Souza ${ }^{(22)}$, no mesmo dia, por dois pesquisadores do estudo, sem que um tomasse conhecimento da avaliação do outro, para evitar um viés na avaliação. Como os resultados das observações foram concordantes entre si, não foi necessária a análise por um terceiro pesquisador. Os escores de cada estação variavam entre quatro pontos (postura correta) e zero (postura totalmente incorreta), sendo o escore total máximo do teste de 24 pontos. Para a análise estatística, foi utilizado o escore total máximo de cada participante.

O questionário informativo objetivou verificar o conhecimento teórico sobre a coluna vertebral ${ }^{(8)}$. Cada questão tinha uma pontuação específica, sendo que o escore máximo a ser atingido era de 18 pontos (quanto maior o escore, melhor o nível de conhecimento teórico). Para a análise do questionário, foi contabilizado o escore máximo de cada participante.
Quadro 1 - Apresentação das etapas do experimento e dos temas das aulas da Escola Postural Adaptada

\begin{tabular}{|c|c|}
\hline Aula & Temas das aulas \\
\hline 1 & $\begin{array}{l}\text { Apresentação e realização do pré-experimento } \\
\text { (foto e questionário) }\end{array}$ \\
\hline 2 & Realização do pré-experimento (filmagem) \\
\hline 3 & $\begin{array}{l}\text { Objetivos da Escola Postural } \\
\text { As curvaturas da coluna vertebral } \\
\text { A posição sentada }\end{array}$ \\
\hline 4 & $\begin{array}{l}\text { Os mecanismos de compensação postural } \\
\text { Permanecer em pé e caminhar corretamente }\end{array}$ \\
\hline 5 & $\begin{array}{l}\text { Estrutura e função dos discos intervertebrais } \\
\text { Sentar e levantar corretamente }\end{array}$ \\
\hline 6 & $\begin{array}{l}\text { Sobrecarga sobre a coluna - início de hérnia } \\
\text { de disco } \\
\text { Agachar, apanhar e levantar objetos corretamente }\end{array}$ \\
\hline 7 & $\begin{array}{l}\text { Sobrecarga sobre a coluna - hérnia de disco } \\
\text { dolorosa } \\
\text { Rotações ou torções da coluna }\end{array}$ \\
\hline 8 & $\begin{array}{l}\text { Posição ideal para dormir e altura do travesseiro } \\
\text { Deitar e levantar corretamente }\end{array}$ \\
\hline 9 & $\begin{array}{l}\text { Musculatura e postura - músculos eretores da } \\
\text { coluna, abdominais e flexores do quadril }\end{array}$ \\
\hline 10 & $\begin{array}{l}\text { Revisão teórica dos conteúdos da Escola } \\
\text { Postural }\end{array}$ \\
\hline 11 & Pós-experimento (foto e questionário) \\
\hline 12 & Pós-experimento (filmagem) \\
\hline $\begin{array}{l}8 \text { meses } \\
\text { após }\end{array}$ & Follow-up (foto, questionário e filmagem) \\
\hline
\end{tabular}

Foram utilizados testes estatísticos não paramétricos: (1) teste de Mann-Whitney, para verificar a diferença entre o GC e o GE, separadamente para as crianças e para os adolescentes no pré-experimento; (2) teste de Wilcoxon, para verificar as diferenças entre pré e pós-experimento, pré-experimento e follow-up e pós-experimento e follow-up, separadamente para as crianças e para os adolescentes. Esses testes foram aplicados na análise das seguintes variáveis: escore total máximo das AVDs e escore máximo do questionário. O teste do qui-quadrado foi empregado para analisar a presença ou a ausência de alterações posturais (frequência de alteração postural) entre pré e pós-experimento, pré-experimento e follow-up e pós-experimento e follow-up, separadamente para as crianças e para os adolescentes. O nível de significância foi de 0,05 . 


\section{Resultados}

No pré-experimento, o GC e o GE apresentaram postura estática semelhante, tanto nas crianças $(p=0,85)$ quanto nos adolescentes $(p=0,69)$.

A comparação da postura estática do GC não evidenciou diferença significativa entre: (1) pré e pós-experimento, tanto para crianças $(p=0,85)$ quanto para adolescentes $(p=0,67)$; (2) pré-experimento e follow-up, tanto para crianças $(p=0,94)$ quanto para adolescentes ( $p=0,91)$; e (3) pós-experimento e follow-up, tanto para crianças ( $p=0,71)$ quanto para adolescentes $(p=0,83)$. Portanto, os participantes do GC, na avaliação inicial e no oitavo mês após a avaliação final, continuaram a apresentar desalinhamentos da postura corporal (Tabela 2).

Do mesmo modo, a comparação da postura estática do GE também não evidenciou diferença significativa entre: (1) pré e pós-experimento, tanto para crianças $(p=0,13)$ quanto para adolescentes $(p=0,07)$ com relação ao número de casos em que ocorreu alguma alteração da postura; (2) pré-experimento e follow-up, tanto para crianças $(p=0,30)$ quanto para adolescentes $(p=0,20)$; e (3) pós-experimento e follow-up, tanto para crianças $(p=0,47)$ como para adolescentes $(p=0,32)$. Pode-se observar que houve uma diminuição do número de pacientes com alterações posturais no GE, ou seja, alguns participantes do PEP apresentaram melhora do seu alinhamento postural (Tabela 1). Já após oito meses do término do PEP, o GE representou um aumento do número de casos com alterações posturais (Tabela 1).

Quanto à postura dinâmica no pré-experimento, não houve diferença significativa entre o GC e o GE de crianças $(p=0,08)$ e de adolescentes $(p=0,66)$. A comparação da postura dinâmica do GC não evidenciou diferença significativa entre pré e pós-experimento ( $p=0,22$ e $p=0,06$, para crianças e adolescentes, respectivamente); pré-experimento e follow-up $(p=0,14$ e $p=0,11$, para crianças e adolescentes, respectivamente); e pós-experimento e follow-up ( $p=0,63$ e $p=0,26$, para crianças e adolescentes, respectivamente). Assim, os participantes do GC continuaram apresentando o mesmo padrão de execução nas AVDs (Tabela 2).

Já a comparação da postura dinâmica do GE evidenciou diferenças entre pré e pós-experimento $(p=0,001$ e $p<0,001$, para crianças e adolescentes, respectivamente) no padrão de execução das AVDs. Tal diferença ocorreu também entre pré-experimento e follow-up ( $p=0,013$ e $p=0,021$, para crianças e adolescentes, respectivamente) e entre pós-experimento e follow-up ( $p=0,008$ e $p=0,002$, para crianças e adolescentes, respectivamente). As diferenças encontradas na execução das AVDs após oito meses do término do PEP, para o GE, mostraram o retorno do padrão inadequado de execução das AVDs, evidenciado na avaliação inicial (Tabela 2).

Tabela 1 - Número de alterações posturais observadas durante a avaliação estática da postura dos participantes do Programa de Educação Postural integrantes dos grupos Controle e Experimental, nos períodos de pré e pós-experimento e follow-up

\begin{tabular}{lcccccc}
\hline \multirow{2}{*}{ Grupo } & \multicolumn{3}{c}{$\mathbf{N}^{\circ}$ de alterações - crianças } & \multicolumn{3}{c}{$\mathbf{N}^{\circ}$ de alterações - adolescentes } \\
\cline { 2 - 7 } & Pré & Pós & Follow-up & Pré & Pós & Follow-up \\
\hline Controle & 15 & 14 & 16 & 12 & 10 & 11 \\
Experimental & 14 & 7 & 10 & 14 & 6 & 10 \\
\hline
\end{tabular}

Tabela 2 - Média e desvio padrão dos escores obtidos no questionário teórico pelos participantes do Programa de Educação Postural integrantes dos grupos Controle e Experimental, nos períodos de pré e pós-experimento e follow-up

\begin{tabular}{lcccccc}
\hline \multirow{2}{*}{ Grupo } & \multicolumn{3}{c}{ Crianças } & \multicolumn{3}{c}{ Adolescentes } \\
\cline { 2 - 7 } & Pré & Pós & Follow-up & Pré & Pós & Follow-up \\
\hline Controle & $4,8 \pm 3,1$ & $5,2 \pm 2,1$ & $5,0 \pm 1,7$ & $6,5 \pm 1,7$ & $6,6 \pm 1,9$ & $6,8 \pm 1,8$ \\
Experimental & $4,7 \pm 2,7$ & $10,9 \pm 1,6$ & $8,0 \pm 1,9$ & $6,6 \pm 1,8$ & $12,4 \pm 3,3$ & $10,1 \pm 2,0$ \\
\hline
\end{tabular}

Tabela 3 - Média e desvio padrão dos escores obtidos na filmagem no circuito de AVDs pelos participantes do Programa de Educação Postural integrantes dos grupos Controle e Experimental, nos períodos de pré e pós-experimento e follow-up

\begin{tabular}{lcccccc}
\hline \multirow{2}{*}{ Grupos } & \multicolumn{3}{c}{ Crianças } & \multicolumn{3}{c}{ Adolescentes } \\
\cline { 2 - 7 } & Pré & Pós & Follow-up & Pré & Pós & Follow-up \\
\hline Controle & $11,8 \pm 3,3$ & $12,2 \pm 2,6$ & $12,0 \pm 3,7$ & $10,5 \pm 3,7$ & $10,6 \pm 2,9$ & $10,8 \pm 3,8$ \\
Experimental & $10,7 \pm 2,7$ & $19,9 \pm 0,6$ & $15,0 \pm 2,6$ & $10,6 \pm 1,8$ & $18,6 \pm 2,4$ & $14,3 \pm 3,5$ \\
\hline
\end{tabular}


O nível de conhecimento teórico sobre a coluna vertebral, no pré-experimento, foi semelhante entre o GC e o GE de crianças $(p=0,32)$ e de adolescentes $(p=0,85)$.

Para o GC, o nível de conhecimento teórico sobre a coluna vertebral não foi diferente significativamente entre pré e pós-experimento ( $p=0,06$ e $p=0,43$, para crianças e adolescentes, respectivamente); pré-experimento e follow-up ( $p=0,12$ e $p=0,08$, para crianças e adolescentes, respectivamente); e pós-experimento e follow-up ( $p=0,26$ e $p=0,23$, para crianças e adolescentes, respectivamente), demonstrando que o GC permaneceu nos três períodos com o mesmo conhecimento teórico sobre a coluna vertebral (Tabela 3).

Já a comparação do nível de conhecimento teórico do GE evidenciou diferenças entre pré e pós-experimento ( $p=0,001$ e $p=0,001$, para crianças e adolescentes, respectivamente); entre pré-experimento e follow-up $(p=0,045$ e $p=0,041$, para crianças e adolescentes, respectivamente) e entre pós-experimento e follow-up ( $p=0,038$ e $p=0,042$, para crianças e adolescentes, respectivamente). As diferenças encontradas no nível de conhecimento teórico após oito meses do término do PEP, para o GE, resultaram em diminuição dos escores obtidos, voltando a apresentar o conhecimento inicial (Tabela 3).

\section{Discussão}

Antes do início do PEP, a avaliação da postura estática evidenciou que praticamente todas as crianças e adolescentes apresentavam algum tipo de alteração postural. Esse fato está em acordo com Rosa Neto ${ }^{(21)}$ e Detsch et al ${ }^{(23)}$, que mostram altas taxas de prevalência de alterações posturais laterais e anteroposteriores na população de estudantes.

Entretanto, observando-se qualitativamente os resultados da Tabela 1 , nota-se que, entre pré e pós-experimento, parece ter ocorrido uma diminuição das alterações da postura de ombro e de cabeça em relação ao fio de prumo, sugerindo uma "mudança postural positiva" provocada pelo PEP para crianças e adolescentes do GE, pois os resultados foram obtidos imediatamente após o término do programa, de modo que as percepções acerca da postura e os novos conceitos trabalhados nas aulas estavam ainda presentes. Já a comparação entre préexperimento e follow-up e entre pós-experimento e follow-up demonstrou também existir uma "mudança postural", mas esta foi no sentido inverso, ou seja, os efeitos positivos apresentados no término do Programa foram anulados, de modo que a postura apresentada antes da Escola Postural retornou (Tabela 1). Esses resultados não foram sustentados pelo tratamento estatístico, uma vez que diferenças significativas não foram encontradas para a frequência de alterações de postura entre as três fases.

Cabe ressaltar que, considerando-se que o período de crescimento possa ter afetado os resultados da postura estática, o presente estudo conta com uma importante limitação, ou seja, a não utilização de nenhum teste específico para avaliar a puberdade, além da idade cronológica. Sendo assim, algumas crianças poderiam estar já na puberdade ou, ainda, alguns adolescentes poderiam não ser púberes. Entretanto, os efeitos dessa limitação ficam amenizados ao se notar que tanto crianças quanto adolescentes apresentaram resultados semelhantes.

Além do processo de crescimento, provavelmente a motivação representa outro fator de influência nos resultados da Escola Postural para a postura estática na fase de pósexperimento e follow- $u p$. Tresca e De Rose ${ }^{(24)}$ e Scalon et al ${ }^{(25)}$ enfatizam que a motivação é dinamizadora da aprendizagem, canalizando as informações percebidas na direção do comportamento, conduzindo os seres humanos à ação ou à inércia, ou seja, é o motivo pelo qual se escolhe fazer ou manter algo. Assim, a motivação foi fundamental para a realização do PEP, pois é decisiva no processo de aprendizagem, sendo responsável pela inércia ou pela ação em qualquer atividade.

É importante salientar que, em nenhum momento, o PEP teve por objetivo corrigir as alterações posturais, pois seu propósito era ensinar as posturas corretas durante as AVDs e a importância de utilizar o slogan "puxe o fio de prumo e sorria" para manter as curvaturas da coluna vertebral durante a AVDs. Nesse sentido, a avaliação da postura estática foi realizada apenas com o objetivo de verificar se os integrantes do GE, após a participação na Escola Postural, buscariam realinhar sua postura estática em função dos conteúdos desenvolvidos nas aulas.

Contrários aos resultados da avaliação de postura estática, quando comparados pré e pós-experimento, os dados da postura dinâmica sugerem que as mudanças positivas ocorridas imediatamente após o término do PEP na execução das AVDs são oriundas dos efeitos da própria Escola Postural, tal como esperado. Méndez e Gómez-Conesa ${ }^{(6)}$ avaliaram o efeito de um programa de educação postural em 106 escolares com nove anos de idade e concluíram que a participação no programa ocasionou melhora do conhecimento teórico e do desempenho nas AVDs realizadas na escola. No Brasil, Ritter ${ }^{(18)}$ desenvolveu uma Escola Postural para 61 escolares com idade média de 15 anos. Para 
a avaliação das AVDs, nas fases de pré e pós-experimento, o autor utilizou o protocolo de Rocha e Souza ${ }^{(22)}$, tal como o presente estudo, notando que os participantes da Escola Postural apresentaram melhora significativa em todas as AVDs, enquanto o grupo controle não modificou seu padrão de realização das AVDs.

Embora seja bem aceito na literatura que as Escolas Posturais tendem a produzir mudanças positivas de comportamento, no que diz respeito à adoção de posturas na execução das AVDs imediatamente após seu término, estudos que visem identificar se essas mudanças permanecem ainda são escassos. No presente estudo, as diferenças encontradas para a postura dinâmica entre pré-experimento e follow-up e entre pós-experimento e follow-up (Tabela 2) são consequência da redução da pontuação do circuito de AVDs, demonstrando que um intervalo de oito meses sem orientação e reforço das novas aprendizagens para uma efetiva incorporação dos hábitos posturais aprendidos na Escola Postural provavelmente deve ter afetado negativamente os efeitos do programa em longo prazo. Assim, o fato de os efeitos do PEP terem sido "passageiros" no presente estudo pode consistir em uma limitação dessa metodologia. Por outro lado, um resultado que estimula a condução de estudos dessa natureza foi encontrado por Cardon et al ${ }^{(13)}$, que verificaram a influência positiva de um programa postural, em médio prazo, em escolares da quarta e quinta séries do ensino fundamental.

Em vista dessas realidades distintas, uma sugestão para fomentar a utilização do PEP, buscando minimizar os efeitos tardios negativos, seria a implantação de "reforços de aprendizagem" periódicos, ou seja, após a participação em programas de educação postural, os participantes seriam convidados a retornar em períodos inferiores a oito meses para vivenciar, por exemplo, em um único encontro, as experiências corporais e posturais desenvolvidas no programa. Weineck ${ }^{(26)}$ refere que pausas na prática de exercícios levam, mesmo após um curto espaço de tempo, a uma lenta extinção dos padrões motores. Assim, pausas longas, sem acompanhamento e/ou vivências dos ensinamentos, conduzem a um desaparecimento progressivo dos padrões motores. Movimentos automatizados se perdem do ponto de vista mecânico, fisiológico e reflexo-condicionado, quando não são renovados de tempo em tempo, o que justifica o retrocesso dos conhecimentos adquiridos com a Escola Postural, quando avaliados no circuito de AVDs no período de follow-up.

O fato de o GE ter apresentado maior número de questões corretas do questionário na comparação dos períodos de pré e pós-experimento em relação às demais comparações (pré-experimento e follow-up; pós-experimento e follow-up) (Tabela 3) também evidencia os efeitos positivos da Escola Postural logo após seu término. Resultado semelhante é demonstrado por Candotti et $a l^{(8)}$ e por Cardon et $a l^{(15)}$, quando crianças que participaram da Escola Postural aprenderam a conhecer e a identificar a coluna vertebral, suas partes e suas funções. Entretanto, no presente estudo, após oito meses de intervalo sem qualquer reforço de ensino nesse período, houve redução do número de acertos nas questões, o que evidencia a necessidade dos "reforços de aprendizagem".

Diante desses resultados, acredita-se ser importante continuar a implantar e a estudar os efeitos de uma Escola Postural, com avaliações em longo prazo, para averiguar a permanência dos conteúdos adquiridos com o programa. Torna-se interessante que novos PEPs sejam realizados com crianças e adolescentes, porém é necessário que, após o término desses programas, haja reforços periódicos por meio de atividades mensais, como palestras e/ou vivências posturais. Essas atividades possivelmente garantiriam o aprendizado e a assimilação dos conteúdos da Escola Postural.

Em suma, os resultados sugerem que o PEP, quando avaliado imediatamente após seu término, promoveu efeito positivo no conhecimento teórico e na postura das AVDs. Porém, esse efeito não foi estendido ao período de follow-up. Portanto, os participantes do PEP, oito meses após seu término, demonstraram: (1) não conhecer e identificar a coluna vertebral, suas partes e funções; e (2) não manter as curvaturas naturais da coluna vertebral, seja durante a postura estática ou durante as AVDs. Tais achados sugerem que o conteúdo teórico e o conhecimento da importância da boa postura durante as AVDs não foram efetivamente incorporados aos hábitos das crianças e dos adolescentes, sinalizando que não houve assimilação dos novos conhecimentos após oito meses do término do programa. 


\section{Referências bibliográficas}

1. Spence SM, Jensen GM, Shepard KF. Comparison of methods of teaching children proper lifting techniques. Phys Ther 1984;64:1055-61.

2. Black A. Escola postural: uma alternativa para a saúde da coluna vertebral. Porto Alegre: Rígel; 1993.

3. Souza JL, Vieira A. Escola postural: um caminho para o conhecimento de si e o bem-estar corporal. Movimento 2003;9:101-22.

4. Cardon GM, De Clercq DL, De Bourdeaudhuij IM. Back education efficacy in elementary schoolchildren: a 1-year follow-up study. Spine (Phila Pa 1976) 2002;27:299-305.

5. Cardon GM, Clercq DL, Geldhof EJ, Verstraete S, Bourdeaudhuij IM. Back education in elementary schoolchildren: the effects of adding a physical activity promotion program to a back care program. Eur Spine J 2007;16:125-33.

6. Méndez FJ, Gómez-Conesa A. Postural hygiene program to prevent low back pain. Spine (Phila Pa 1976) 2001;26:1280-6.

7. Robertson HC, Lee V. Effects of back care lessons on sitting and lifting by primary students. Aust J Physiother 1990;36:245-8.

8. Candotti CT, Macedo $\mathrm{CH}$, Noll M, Freitas K. Postural school: a methodology adapted for children. Rev Arq Movimento [serial on the Internet]. 2009;5(2):3449 [cited 2010 Nov 5]. Available from: http://vomer2.eefd.ufrj.br/ revista/index. php/EEFD/article/view/38/58

9. Brox JI, Storheim K, Grotle M, Tveito TH, Indahl A, Eriksen HR. Systematic review of back schools, brief education, and fear-avoidance training for chronic low back pain. Spine J 2008;8:948-58.

10. Ferreira MS, Navega MT. Efeitos de um programa de orientação para adultos com lombalgia. Acta Ortop Bras 2010;18:127-31.

11. Rebolho MC, Casarotto RA, João SM. Strategies for teaching postural habits to children: comic strips vs. practical experience. Fisioter Pesq 2009;16:46-51.

12. Martelli RC, Traebert J. Descriptive study of backbone postural changes in 10 to 16 year-old schoolchildren. Tangará-SC, Brazil, 2004. Rev Bras Epidemiol 2006;9:87-93.

13. Cardon G, De Bourdeaudhuij I, De Clercq D. Back care education in elementary school: a pilot study investigating the complementary role of the class teacher. Patient Educ Couns 2001;45:219-26.

14. Zapater AR, Silveira DM, Vitta A, Padovani CR, Silva JC. Seat posture: the efficiency of an educational program for scholars. Cienc Saude Coletiva 2004;9:191-9.

15. Cardon G, De Clercq D, De Bourdeaudhuij I. Effects of back care education in elementary schoolchildren. Acta Paediatr 2000;89:1010-7.

16. Vieira A, Souza JL. Boa postura: uma preocupação com a estética, a mora ou a saúde? Movimento 2009;15:145-65.

17. Steele EJ, Dawson AP, Hiller JE. School-based interventions for spinal pain: a systematic review. Spine (Phila Pa 1976) 2006;31:226-33.

18. Ritter AL. Programa postural para escolares do ensino fundamental [tese de mestrado]. Porto Alegre (RS): UFRGS; 2003.

19. Souza JL. Efeitos de uma Escola Postural para indivíduos com dores nas costas. Movimento 1996;3:56-71.

20. Kendall FP, McCreary EK, Provance PG. Músculos: provas e funções. $4^{\mathrm{a}}$ ed. São Paulo: Manole; 1995.

21. Rosa Neto F. Avaliação postural em escolares de $1^{\mathrm{a}}$ a $4^{\mathrm{a}}$ série do $1^{\circ} \mathrm{grau}$ Rev Bras Cienc Mov 1991;5:7-11.

22. Rocha AS, Souza JL. Observation of daily chores through video. Movimento 1999;5:16-22.

23. Detsch C, Luz AM, Candotti CT, Oliveira DS, Lazaron F, Guimarães LK et al Prevalência de alterações posturais em escolares do ensino médio em uma cidade no Sul do Brasil. Rev Panam Salud Publica 2007;21:231-8.

24. Tresca RP, De Rose Jr D. Estudo comparativo da motivação intrínseca em escolares praticantes e não praticantes de dança. Rev Bras Cienc Mov 2000;8:9-13.

25. Scalon RM, Becker B, Brauner MR. Fatores motivacionais que influenciam na aderência dos programas de iniciação desportiva pela criança. Perfil 1999;1:10-5.

26. Weineck J. Biologia do esporte. $7^{a}$ ed. São Paulo: Manole; 2005. 